\title{
Oscillation Criteria for a Class of Certain Half-linear Emden-Fowler Functional Differential Equations of Neutral Type
}

\author{
Ying TANG and Lian-Zhong LI
}

School of science, Jiangnan university, wuxi 214122, China

820148700@qq.com; Ilz3497@163.com

Keywords: Emden-Fowler equation; half-linear differential equation; Oscillation criterion.

Abstract: Some oscillation criteria for the half-linear Emden-Fowler functional differential equations of neutral type of the form

$$
\left(r(t)\left|z^{\prime}(t)\right|^{\alpha-1} z^{\prime}(t)\right)^{\prime}+q(t)|x(\sigma(t))|^{\beta-1} x(\sigma(t))=e(t), t \geq t_{0}
$$

are established, where $z(t)=x(t)+p(t) x(\tau(t)), \quad \alpha>0, \beta>0$ are arbitrary constants, some earlier results are the special cases of ours since the function $e(t)$ is introduced to the equation.

\section{Introduction}

This paper consider half-linear Emden-Fowler functional differential equations of neutral type

$$
\left(r(t)\left|z^{\prime}(t)\right|^{\alpha-1} z^{\prime}(t)\right)^{\prime}+q(t)|x(\sigma(t))|^{\beta-1} x(\sigma(t))=e(t), t \geq t_{0}
$$

where $z(t)=x(t)+p(t) x(\tau(t)), \alpha>0, \beta>0$.

The following conditions are always valid, if not stated.

$$
\begin{array}{ll}
\left(A_{1}\right) & r(t) \in C^{1}\left(\left[\mathrm{t}_{0}, \infty\right), R\right), r(t)>0, r^{\prime}(t) \geq 0 . \\
\left(A_{2}\right) & p(t), q(t) \in C\left(\left[\mathrm{t}_{0}, \infty\right), R\right), 0 \leq p(t) \leq 1, q(t) \geq 0 . \\
\left(A_{3}\right) & \tau(t) \in C\left(\left[\mathrm{t}_{0}, \infty\right), R\right), \tau(t) \leq t, \lim _{t \rightarrow \infty} \tau(t)=\infty . \\
\left(A_{4}\right) & \sigma(t) \in C^{1}\left(\left[\mathrm{t}_{0}, \infty\right), R\right), \sigma(t)>0, \sigma^{\prime}(t)>0, \sigma(t) \leq t, \lim _{t \rightarrow \infty} \sigma(t)=\infty . \\
\left(A_{5}\right) & e(t) \in C^{1}\left(\left[\mathrm{t}_{0}, \infty\right), R\right), e(t) \leq 0 .
\end{array}
$$

\section{Definition}

The function $x(t) \in C^{1}\left(\left[T_{x}, \infty\right), R\right), T_{x} \geq t_{0}$, is called a solution of the equation (1.1), if it has the properties of $r(t)\left|z^{\prime}(t)\right|^{\alpha-1} z^{\prime}(t) \in C^{1}\left(\left[T_{x}, \infty\right), R\right)$, and it satisfies the equation in $\left[T_{x}, \infty\right)$, moreover if it has arbitrarily large zeros for $T \geq T_{x}$, then we call $x(t)$ is a oscillatory solution of equation (1.1), otherwise it is called nonoscillatory. If each solution of (1.1) is oscillatory, we call equation (1.1) is oscillatory. This paper only consider the non-trivial solution of equation (1.1).

\section{Main Results}

Theorem 2.1 Assume that $\int_{\mathrm{t}_{0}}^{\infty}\left(\frac{1}{r(t)}\right)^{\frac{1}{\alpha}} d t=\infty$, if there exists a function $\rho(t) \in C^{1}\left(\left[T_{0}, \infty\right),(0, \infty)\right)$, such that for any $M>0$ and $m \in(0,1)$, 
$\int_{t_{0}}^{\infty}\left[\rho(t) \bar{p}(t)-\left|\frac{\rho^{\prime}(t)}{\rho(t)}+\mathrm{Me}(t)\right|^{\lambda+1} \frac{r(\lambda(t))}{(\lambda+1)^{\lambda+1}}\left(\frac{\rho(t)}{m \sigma^{\prime}(t)}\right)^{\lambda}\right] d t=\infty$

where $\bar{p}(t)=q(t)(1-p(\sigma(t)))^{\beta}, \lambda=\min \{\alpha, \beta\}$,

$$
\lambda(t)=\left\{\begin{array}{c}
\sigma(t), \beta \geq \alpha \\
t, \alpha>\beta
\end{array}, m=\left\{\begin{array}{c}
1, \quad \alpha=\beta, \\
0<m \leq 1, \alpha \neq \beta .
\end{array}\right.\right.
$$

Then Eq. (1.1) is oscillatory.

Proof. Suppose that Eq. (1.1) has a nonoscillatory solution $x(t)$, without loss of generality, we assume that $x(t)>0$.

From $([6](2.7))$ we have

$$
\left(r(t)\left(z^{\prime}(t)\right)^{\alpha}\right)^{\prime}+\bar{p}(t) z^{\beta}(\sigma(t)) \leq 0,
$$

which follows that

$\left(r(t)\left(z^{\prime}(t)\right)^{\alpha}\right)^{\prime}+\bar{p}(t) z^{\beta}(\sigma(t)) \leq e(t)$

where $\bar{p}(t)=q(t)[1-p(\sigma(t))]^{\beta}$,

then we have

$$
\bar{p}(t) z^{\beta}(\sigma(t)) \leq e(t)-\left[r(t)\left(z^{\prime}(t)\right)^{\alpha}\right]^{\prime} .
$$

Define

$$
w(t)=\rho(t) \frac{r(t)\left(z^{\prime}(t)\right)^{\alpha}}{z^{\beta}(\sigma(t))}
$$

Obviously, $w(t)>0$, and $r(t)\left(z^{\prime}(t)\right)^{\alpha}$ is nonincreasing,

$$
\begin{aligned}
& w^{\prime}(t)=\rho^{\prime}(t) \frac{w(t)}{\rho(t)}+\rho(t) \frac{\left[r(t)\left(z^{\prime}(t)\right)^{\alpha}\right]^{\prime}}{z^{\beta}(\sigma(t))}-\rho(t) \frac{\left[r(t)\left(z^{\prime}(t)\right)^{\alpha}\right] \beta z^{\beta-1}(\sigma(t)) z^{\prime}(\sigma(t)) \sigma^{\prime}(t)}{z^{2 \beta}(\sigma(t))} \\
& \leq-\rho(t) \bar{p}(t)+\rho^{\prime}(t) \frac{w(t)}{\rho(t)}+\frac{e(t) w(t)}{r(t)\left(z^{\prime}(t)\right)^{\alpha}}-\rho(t) \frac{\left[r(t)\left(z^{\prime}(t)\right)^{\alpha}\right] \beta z^{\beta-1}(\sigma(t)) z^{\prime}(\sigma(t)) \sigma^{\prime}(t)}{z^{2 \beta}(\sigma(t))} .
\end{aligned}
$$

which follows that

$$
w^{\prime}(t) \leq-\rho(t) \bar{p}(t)+\left[\frac{\rho^{\prime}(t)}{\rho(t)}+\frac{e(t)}{r(t)\left(z^{\prime}(t)\right)^{\alpha}}\right] w(t)-\frac{\beta \sigma^{\prime}(t)}{(\rho(t) r(\sigma(t)))^{\frac{1}{\alpha}}}[z(\sigma(t))]^{\frac{\beta-\alpha}{\alpha}} w^{\frac{\alpha+1}{\alpha}}(t), \quad \text { (2.6) }
$$

since $e(t) \leq 0$, there exits $M>0$ such that,

$$
r(t)\left(z^{\prime}(t)\right)^{\alpha} \leq \frac{1}{M}
$$




$$
\frac{e(t)}{r(t)\left(z^{\prime}(t)\right)^{\alpha}} \leq M e(t)
$$

which follows that

$$
\begin{aligned}
& w^{\prime}(t) \leq-\rho(t) \bar{p}(t)+\left[\frac{\rho^{\prime}(t)}{\rho(t)}+\mathrm{Me}(t)\right] w(t)-\frac{\beta \sigma^{\prime}(t)}{(\rho(t) r(t))^{\frac{1}{\alpha}}}[z(\sigma(t))]^{\frac{\beta-\alpha}{\alpha}} w^{\frac{\alpha+1}{\alpha}}(t) \\
\leq & -\rho(t) \bar{p}(t)+\left|\frac{\rho^{\prime}(t)}{\rho(t)}+\operatorname{Me}(t)\right| w(t)-\frac{\beta \sigma^{\prime}(t)}{(\rho(t) r(t))^{\frac{1}{\alpha}}}[z(\sigma(t))]^{\frac{\beta-\alpha}{\alpha}} w^{\frac{\alpha+1}{\alpha}}(t) .
\end{aligned}
$$

$z^{\prime}(t)$ is a monotone increasing function, there exists a constant $m \in(0,1)$, such that

$$
[z(\sigma(t))]^{\frac{\beta-\alpha}{\alpha}} \geq m
$$

Then we have

$$
w^{\prime}(t) \leq-\rho(t) \bar{p}(t)+\left|\frac{\rho^{\prime}(t)}{\rho(t)}+\mathrm{Me}(t)\right| w(t)-\frac{m \beta \sigma^{\prime}(t)}{(\rho(t) r(\sigma(t)))^{\frac{1}{\alpha}}} w^{\frac{\alpha+1}{\alpha}}(t) .
$$

Considering

$$
A x-B x^{\frac{\lambda+1}{\lambda}} \leq \frac{\lambda^{\lambda}}{(\lambda+1)^{\lambda+1}} A^{\lambda+1} B^{-\lambda}, \text { where } B>0, A \geq 0, x \geq 0,
$$

then

$$
\begin{aligned}
w^{\prime}(t) & \leq-\rho(t) \bar{p}(t)+\frac{\lambda^{\lambda}}{(\lambda+1)^{\lambda+1}}\left|\frac{\rho^{\prime}(t)}{\rho(t)}+\operatorname{Me}(t)\right|^{\lambda+1}\left(\frac{\lambda m \sigma^{\prime}(t)}{(\rho(t) r(\sigma(t)))^{\frac{1}{\alpha}}}\right)^{-\lambda} \\
& =-\rho(t) \bar{p}(t)+\left|\frac{\rho^{\prime}(t)}{\rho(t)}+\operatorname{Me}(t)\right|^{\lambda+1} \frac{r(\lambda(t))}{(\lambda+1)^{\lambda+1}}\left(\frac{m \sigma^{\prime}(t)}{\rho(t)}\right)^{-\lambda} \\
& =-\left\{\rho(t) \bar{p}(t)-\left|\frac{\rho^{\prime}(t)}{\rho(t)}+\operatorname{Me}(t)\right|^{\lambda+1} \frac{r(\lambda(t))}{(\lambda+1)^{\lambda+1}}\left(\frac{\rho(t)}{m \sigma^{\prime}(t)}\right)^{\lambda}\right\} .
\end{aligned}
$$

Integrating the above inequality from $\mathrm{T}$ to $\mathrm{t}$, we have

$$
w(t) \leq w(T)-\int_{T}^{t}\left\{\rho(s) \bar{p}(s)-\left|\frac{\rho^{\prime}(s)}{\rho(s)}+\mathrm{Me}(s)\right|^{\lambda+1} \frac{r(\lambda(s))}{(\lambda+1)^{\lambda+1}}\left(\frac{\rho(s)}{m \sigma^{\prime}(s)}\right)^{\lambda}\right\} d s,
$$

letting $t \rightarrow \infty$, we have $w(t) \rightarrow-\infty$, which is a contradiction. This completes the proof of Theorem 2.1.

Corollary 2.1 Assume that $e(t)=0$ and(2.1)hold, other conditions unchanged,

$$
\int_{t_{0}}^{\infty}\left[\rho(t) \bar{p}(t)-\left|\frac{\rho^{\prime}(t)}{\rho(t)}\right|^{\lambda+1} \frac{r(\lambda(t))}{(\lambda+1)^{\lambda+1}}\left(\frac{\rho(t)}{m \sigma^{\prime}(t)}\right)^{\lambda}\right] d t=\infty .
$$

then Eq. (1.1) is oscillatory.

Corollary 2.2 Assume that $e(t)=0$ and $\alpha=\beta$, 


$$
\int_{t_{0}}^{\infty}\left[\rho(t) \bar{p}(t)-\left|\frac{\rho^{\prime}(t)}{\rho(t)}\right|^{\alpha+1} \frac{r(\sigma(t))}{(\alpha+1)^{\alpha+1}}\left(\frac{\rho(t)}{\sigma^{\prime}(t)}\right)^{\alpha}\right] d t=\infty
$$

(2.1)is replaced by (2.12), then Eq. (1.1) is oscillatory.

Corollary 2.3 Assume that $\alpha=1$, condition(2.1)in Theorem 2.1 is replaced by one of the following form:

(1) If $\beta>1$,

$$
\int_{t_{0}}^{\infty}\left[\rho(t) \bar{p}(t)-\left|\frac{\rho^{\prime}(t)}{\rho(t)}+M e(t)\right|^{2} \frac{r(\sigma(t)) \rho(t)}{4 m \sigma^{\prime}(t)}\right] d t=\infty ;
$$

(2) If $0<\beta<1$,

$$
\int_{t_{0}}^{\infty}\left[\rho(t) \bar{p}(t)-\left|\frac{\rho^{\prime}(t)}{\rho(t)}+M e(t)\right|^{\beta+1} \frac{r(t)}{(\beta+1)^{\beta+1}}\left(\frac{\rho(t)}{m \sigma^{\prime}(t)}\right)^{\beta+1}\right] d t=\infty .
$$

The conclusion of theorem 2.1 is still true.

\section{References}

[1] G.H.Hardy,J.E.Littlewood and G.Polya. Inequalities, second edition. Cambrige Univercity Press, Cambrige, 1988.

[2] Leighton W.The detection of the oscillations of solutions of a second orderlinear differential equation.Duke Math J,1950,17:57-62.

[3] Grammatikopoulos M K,Ladas G,Meimaridou A.Oscillations of second order neutral delay differential equations.Rad Math,1985,1:267-274.

[4] Sun Y G,Meng F W. Note on the paper of Dzurina and Stavroulakis.Appl Math Comput,2006,174:1634-1641.

[5] T.X.Li, Z.L.Han,C.H.Zhang and S.R.Sun. On the oscillation of second order Emden-Fowler neutral differential equations. J. Appl Math Comput, 37(2011):601-610.

[6] Liu H D,Meng F W,Liu P H.Oscillation and asymptotic analysis on a new generalized Emden-Fowler equation.Appl Math Comput,2012, 219:2739-2748.

[7] Ch.G.Philos, Oscillation theorems for linear differential equations of second order, Arch. Math (Basel).53(1989):482-492.

[8] Agarwal R P,Shieh S L,Yeh C C.Oscillation criteria for second order retarded differential equations.Math comput Modelling,1997,26:1-11.

[9] Baculikova B,Li T,Dzurina J.Oscillation theorems for second order super-linear neutral differential equations.MathematicaSlovaca, 2013,63:123-134.

[10] Dong J G.Oscillation behavior of second-order nonlinear neutral differential equations with deviating arguments.comput Math Appl, 2010,59:3710-3717.

[11] Erbe L,Hassan T S,Peterson A. Oscillation of second-order neutral differential equations.Adv Dynam Syst Appl,2008,3:53-71.

[12] Liu L,Bai Y.New oscillation criteria for second order nonlinear delay neutral differential equations.J Comput Appl Math,2009,231:657-663. 